\title{
Association of the PTEN IVS4 (rs3830675) Gene Polymorphism with Reduced Risk of Cancer: Evidence from a Meta-analysis
}

\author{
Raju K Mandal ${ }^{1}$, Naseem Akhter ${ }^{2}$, Mohammad Irshad ${ }^{2}$, Aditya K Panda ${ }^{3}$, Arif \\ Ali $^{2}$, Shafiul Haque ${ }^{1,2 *}$
}

\begin{abstract}
PTEN (phosphatase and tensin homologue), as a tumor suppressor gene, plays a significant role in regulating cell growth, proliferation, and apoptosis. Results from published studies for association between the PTEN IVS4 I/D (rs3830675) polymorphism and cancer risk are inconsistent and inconclusive. We therefore conducted a meta-analysis to evaluate the potential association between PTEN IVS4 I/D polymorphism and risk of cancer in detail. We searched PubMed (Medline) and EMBASE web databases to cover all relevant studies published until December 2013. The meta-analysis was carried out and pooled odds ratios (ORs) and $95 \%$ confidence intervals $(95 \% \mathrm{CIs})$ were used to appraise the strength of association. A total of 1,993 confirmed cancer cases and 3,200 controls were included from six eligible case-control studies. Results from overall pooled analysis suggested a significant effect of the PTEN IVS4 I/D polymorphism and cancer risk in all genetic models, i.e., allele (I $v$ s D: OR=0.743, 95\% CI=0.648 to 0.852 , $\mathrm{p}=0.001$ ), homozygous (II $v s$ DD: $\mathrm{OR}=0.673,95 \% \mathrm{CI}=0.555$ to $0.816, p=0.001$ ), heterozygous (ID vs DD: $\mathrm{OR}=0.641,95 \% \mathrm{CI}=0.489$ to $0.840, \mathrm{p}=0.001$ ), dominant (II+ID vs DD: $\mathrm{OR}=0.626,95 \% \mathrm{CI}=0.489$ to $0.802, \mathrm{p}=0.001$ ) and recessive (II $v s \mathrm{DD}+\mathrm{ID}$ : $\mathrm{OR}=\mathbf{0 . 7 4 9 ,}, 95 \% \mathrm{CI}=0.631$ to 0.889 , $\mathrm{p}=0.001$ ). Significant publication bias was detected during the analysis. The present meta-analysis suggests that the PTEN IVS4 I/D polymorphism is significantly associated with reduced risk of cancer. However, future larger studies with other groups of populations are warranted to clarify this association.
\end{abstract}

Keywords: Meta-analysis - PTEN - cancer - polymorphism - susceptibility

Asian Pac J Cancer Prev, 16 (3), 897-902

\section{Introduction}

Cancer is a polygenic disease, which results from complex interactions between environmental and genetic factors (Pharoah et al., 2004). It remains a major global health problem and leads to increase morbidity and mortality worldwide (Jemal et al., 2011). The precise etiology of this fatal disease is still unclear. Its increasing incidence and mortality rate during the last two decades has posed a big challenge to clinicians and scientists. Epidemiological studies continue to indicate the significant contribution of 'single nucleotide polymorphisms (SNPs)' to cancer susceptibility through interaction with various environmental factors (Lichtenstein et al., 2000). Thus, it can be anticipated that the discovery of genetic factors of host for cancer risk would assist in the therapeutic applications and overall control of this lethal disease.

The PTEN (Phosphatase and tensin homolog, also known as MMAC1, MIM no. 601728) is a well studied tumor suppressor gene mapped on human chromosome 10q23 (Li et al., 1997). It encodes a 403 amino acid protein homologous to some protein phosphatases, and the protein has been shown to possess protein phosphatase activity in-vitro (Steck et al., 1997) Genetic alternation at PTEN locus has been described in various cancers (Li et al., 1997). PTEN plays an important role in the modulation of phosphatidylinositol 3-kinase pathway $(\mathrm{PI} 3 \mathrm{~K})$ that is involved in cell proliferation and survival in colorectal cancer, gastric cancer, renal clear cell carcinomas (RCCC) (Besson et al., 1999; Cheng et al, 2012; Pandurangan, 2013; Wen- Ting et al., 2014; Wu et al., 1998). Abnormalities in the PI3K pathway are involved in the pathogenesis of different type of cancers. An early study showed that genetic variants in the PI3K/ PTEN/AKT/mTOR pathway may predict platinum-based chemotherapy response in advanced Non-small Cell Lung Cancers (NSCLC) patients in a Chinese population (Jia- Li et al., 2012). Also, PTEN regulates cell cycle progression and cell survival (Tamura et al., 1998; Tamura et al., 1999). Mutation in PTEN gene revealed an increased growth rate in embryonic stem cells and exhibited an advanced entry in to S-phase of the cell cycle (Sun et al., 1999). Many

${ }^{1}$ Research and Scientific Studies Unit, College of Nursing and Allied Health Sciences, Jazan University, Jazan, Saudi Arabia, ${ }^{2}$ Department of Biosciences, Jamia Millia Islamia (A Central University), New Delhi, ${ }^{3}$ Department of Infectious Disease Biology, Institute of Life Sciences, Bhubaneswar, Odisha, India*For correspondence: shafiul.haque@hotmail.com 
SNPs have been reported in PTEN gene, among them IVS4 (rs3830675) occurring with ACTAA insertion at the 109bp downstream of exon 4 in intron 4 is one of the common SNP in PTEN gene (Carroll et al., 1999).

Keeping the significant role of PTEN in carcinogenesis in view, several molecular epidemiological case-control studies have been performed to examine the possible association between the PTEN IVS4 I/D polymorphism and human malignancy in different populations (Ozturk et al., 2013; Canbay et al., 2013a; Canbay et al., 2013b; Ge et al., 2008; Onay et al., 2006; George et al., 2001), but none has been found conclusive. Inconsistency in the results of these studies could possibly be attributed to ethnicity of the population, sample size from individual studies that have low power to evaluate the overall effect. Therefore, it was necessary to quantify and summarize results from all eligible studies with rigorous statistical methods for precise conclusion. In the present study, we performed meta-analysis to evaluate the overall association of IVS4 I/D polymorphism in risk/resistance to the development of cancer. A meta-analysis employs quantitative approach to combine the data from individual studies where individual sample sizes are small with lower statistical power, and gives precise conclusion (Cohn and Becker, 2003; Mandal et al., 2013).

\section{Materials and Methods}

\section{Identification and eligibility of relevant studies}

We searched research literature from electronic data bases, viz., PubMed (Medline) and EMBASE, last search updated on December 2013 with the combination of following keywords: 'PTEN IVS4 I/D and/or (rs3830675) (polymorphism OR mutation OR variant) AND cancer susceptibility or risk. The search was focused on studies that had been carried out in humans. All retrieved articles were evaluated by reading the titles and abstracts, and all published studies matching with the pre-set eligible criteria were retrieved for this meta-analysis. We also did manual search of reference list from the retrieved articles for other eligible studies.

\section{Inclusion and exclusion criteria}

Articles included in the current meta-analysis had to meet all the following criteria: i) must have evaluated the association between PTEN IVS4 polymorphism and cancer risk, ii) used a case-control design, iii) recruited histologically confirmed cancer patients and healthy controls, iv) have available genotype frequency in case and control, v) must be published in the English language. In addition to above, when the same patient population was included in more than one publication, only the most recent or complete study was included in this metaanalysis. The most important reasons for study exclusion were, overlapping of the data, case-only studies, review articles, and genotype frequencies or number not reported. The information regarding the selection of the studies is provided as a flow diagram in Figure 1.

\section{Data extraction and quality assessment}

For each retrieved research article, the methodological quality assessment and data extraction were independently abstracted in duplicate by two independent investigators using a standard protocol. Standard data-collection form was used to ensure the accuracy of the collected data by strictly following the inclusion-exclusion criteria mentioned above. The major characteristics abstracted from the retrieved studies included the name of the first author, year of publication, the country of origin, the number of cases and controls, type of cancer, genotype frequencies for cases and controls and source of genotyping. Cases related with disagreement on any item of the data from the collected studies were fully discussed with the investigators to achieve a final consensus.

\section{Statistical analysis}

In order to determine the strength of the association between PTEN IVS4 I/D polymorphism and cancer risk, the pooled ORs and their corresponding 95\%CIs were calculated (Woolf, 1995). Heterogeneity assumption between studies across the eligible comparison was done by the chi-square based Q-test (Wu and Li, 1999). Heterogeneity was considered significant at p-value $<0.05$, to avoid underestimation of the presence of heterogeneity. A fixed effect model (if $\mathrm{p}>0.05$ ) (Mantel and Haenszel, 1959) or a random effect model (if $\mathrm{p}$ $<0.05$ ) (DerSimonian and Laird, 1986) were used for pooling the results. Moreover, $\mathrm{I}^{2}$ statistics was employed to efficiently test the heterogeneity (Higgins et al., 2003). Hardy-Weinberg equilibrium (HWE) in the controls was measured via chi-square test. Funnel plot asymmetry was estimated by Egger's linear regression test (a type of linear regression approach for the estimation of funnel plot asymmetry on the natural logarithm scale of the OR). The significance of the intercept was determined by the t-test (p-value $<0.05$ was considered as representation of statistically significant publication bias level) (Egger et al., 1997). The statistical analysis involved in this metaanalysis was performed by Comprehensive Meta-Analysis

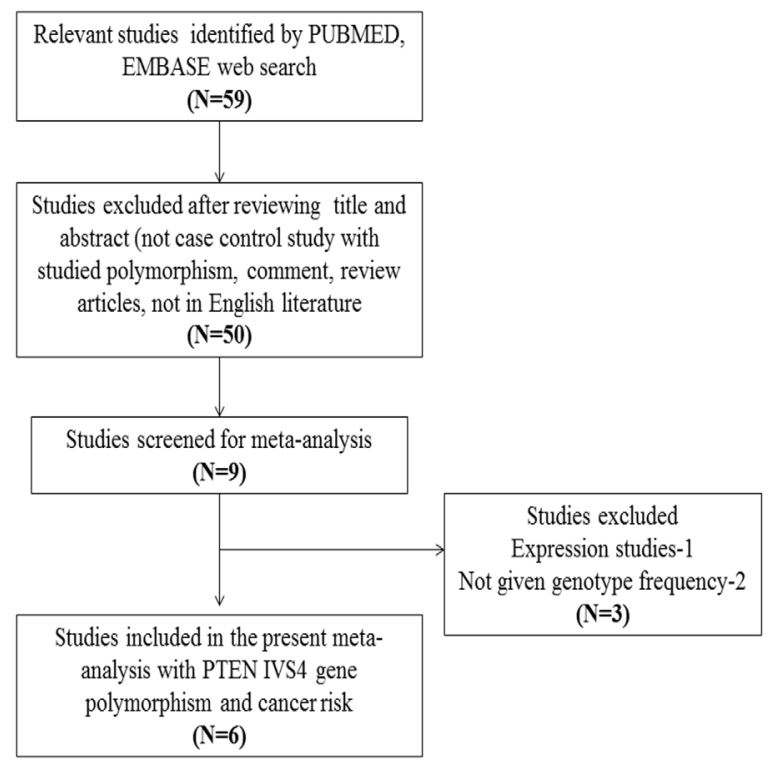

Figure 1. Flow Chart Showing the Identification and Selection (Inclusion/Exclusion Criteria) of the Studies for the Present Meta-analysis 
(CMA) Version 2 software (Biostat Inc., NJ, USA). All p-values were two sided and $p$-value $<0.05$ was considered as statistical significance level.

\section{Results}

Literature search and meta-analysis databases

As per the pre-set selection (inclusion-exclusion) criteria, a total number of six research articles were finally included through literature search from the PubMed (Medline) and EMBASE in this meta-analysis. All retrieved articles were examined carefully by reading the titles and abstracts, and the full texts for the potentially pertinent publications were further checked for their suitability for this meta-analysis. Studies either showing PTEN polymorphism to predict survival in cancer patients or considering PTEN variants as an indicators for response to therapy were excluded straightaway. Similarly, research articles investigating the levels of PTEN mRNA or protein expression or relevant review articles were also excluded. We included only case-control or cohort design studies having frequency of all three genotype (Figure 1). Besides the database search, the references listed in the retrieved publications were also checked for other potential studies (Table 1). Distributions of genotypes and minor allele frequency (MAF) in the controls and cases have been given in Table 2.

\section{Publication bias}

The Begg's funnel plot and Egger's test were performed to examine the publication bias among the included studies for this meta-analysis. The appearance of the shape of funnel plots and the results of Egger's test have shown the evidence of publication bias in four genetic models (Table 3 ). The trim and fill method was employed to adjust the publication bias for recomputing the effect size (Duval and Tweedie, 2000).

\section{Evaluation of heterogeneity}

In order to test heterogeneity among the selected studies, Q-test and $\mathrm{I}^{2}$ statistics were employed. Heterogeneity was observed in three genetic models, i.e., allele (I vs D), heterozygous (ID $v s$ DD) and recessive (II+ID vs DD). Thus, random effects model was applied to synthesize the data for above three models (Table 3 ).

\section{Association of PTEN IVS4 polymorphism and overall cancer susceptibility}

We pooled all six studies together which resulted into 1993 confirmed cancer cases and 3200 healthy

\section{Table 1. Case-Control Studies Included in the Present Meta-analysis}

\begin{tabular}{llllll}
\hline $\begin{array}{l}\text { First authors, publication } \\
\text { year }\end{array}$ & Country & Types of cancer & Study design & Genotyping method & Controls \\
\hline Ozturk et al. 2013 & Turkey & Breast & HB & PCR-RFLP & 128 \\
Canbay et al. 2013 & Turkey & Colon & HB & PCR-RFLP & 118 \\
Canbay et al. 2013 & Turkey & Rectum & HB & PCR-RFLP & 75 \\
Canbay et al. 2013 & Turkey & Gastric & HB & PCR-RFLP & PB \\
Ge et al. 2008 & China & Esophageal & HB, PB & PCR-RFLP & 634 \\
Ge et al. 2008 & China & Gastric & HB, PB & PCR-RFLP & 634 \\
Onay et al. 2006 & Canada & Breast & HB, PB & TaqMan & 350 \\
George et al. 2001 & USA & Prostate & PB & PCR-RFLP & 398 \\
\hline
\end{tabular}

*HB: Hospital based; PB: Population based; PCR-RFLP: Polymerase chain reaction- Restriction fragment length polymorphism; TaqMan: Realtime PCR chemistry

Table 2. Genotypic Distribution of PTEN IVS4 I/D Gene Polymorphism of All Eligible Studies Included in the Present Meta-analysis

\begin{tabular}{|c|c|c|c|c|c|c|c|c|c|}
\hline \multirow[t]{3}{*}{ Authors and year } & \multicolumn{4}{|c|}{ Controls } & \multicolumn{4}{|c|}{ Cancer cases } & \multirow{3}{*}{$\begin{array}{c}\text { HWE } \\
\text { p-value }\end{array}$} \\
\hline & \multicolumn{3}{|c|}{ Genotype } & \multirow{2}{*}{$\begin{array}{c}\text { Minor allele } \\
\text { MAF }\end{array}$} & \multicolumn{3}{|c|}{ Genotype } & \multirow{2}{*}{$\begin{array}{c}\text { Minor allele } \\
\text { MAF }\end{array}$} & \\
\hline & DD & ID & II & & DD & ID & II & & \\
\hline Ozturk et al. 2013 & 41 & 79 & 8 & 0.37 & 57 & 55 & 6 & 0.28 & 0.0002 \\
\hline Canbay et al. 2013 & 86 & 144 & 15 & 0.35 & 74 & 47 & 6 & 0.23 & $<0.0001$ \\
\hline Canbay et al. 2013 & 86 & 144 & 15 & 0.35 & 41 & 34 & 1 & 0.23 & $<0.0001$ \\
\hline Canbay et al. 2013 & 43 & 60 & 10 & 0.35 & 57 & 29 & 7 & 0.23 & 0.08 \\
\hline Ge et al. 2008 & 138 & 329 & 167 & 0.52 & 94 & 183 & 73 & 0.47 & 0.31 \\
\hline Ge et al. 2008 & 138 & 329 & 167 & 0.52 & 63 & 146 & 48 & 0.47 & 0.31 \\
\hline Onay et al. 2006 & 171 & 180 & 47 & 0.34 & 196 & 138 & 38 & 0.28 & 0.97 \\
\hline George et al. 2001 & 346 & 367 & 90 & 0.34 & 262 & 281 & 57 & 0.32 & 0.62 \\
\hline
\end{tabular}

Table 3. Statistics to Test Publication Bias and Heterogeneity in the Present Meta-analysis

\begin{tabular}{|c|c|c|c|c|c|c|c|}
\hline \multirow[t]{2}{*}{ Comparisons } & \multicolumn{3}{|c|}{ Egger's regression analysis } & \multicolumn{3}{|c|}{ Heterogeneity analysis } & \multirow{2}{*}{$\begin{array}{l}\text { Model used for } \\
\text { the meta-analysis }\end{array}$} \\
\hline & Intercept & 95\% Confidence Interval & $\overline{\text { p-value }}$ & $\overline{\mathrm{Q}-\mathrm{value}}$ & Pheterogeneity & $\overline{\mathrm{I}^{2}(\%)}$ & \\
\hline I $v s \mathrm{D}$ & -3.72 & -5.19 to -2.25 & 0.008 & 15.52 & 0.03 & 54.92 & Random \\
\hline II $v s \mathrm{DD}$ & -1.36 & -2.34 to -0.38 & 0.01 & 4.64 & 0.70 & $<0.0001$ & Fixed \\
\hline ID $v s$ DD & -5.15 & -8.25 to -2.05 & 0.006 & 28.69 & $<0.0001$ & 75.60 & Random \\
\hline $\mathrm{II}+\mathrm{ID} v s \mathrm{DD}$ & -4.99 & -7.51 to -2.47 & 0.002 & 25.99 & 0.001 & 73.06 & Random \\
\hline II $v s \mathrm{DD}+\mathrm{ID}$ & -0.36 & -1.57 to 0.84 & 0.49 & 2.97 & 0.88 & $<0.0001$ & Fixed \\
\hline
\end{tabular}




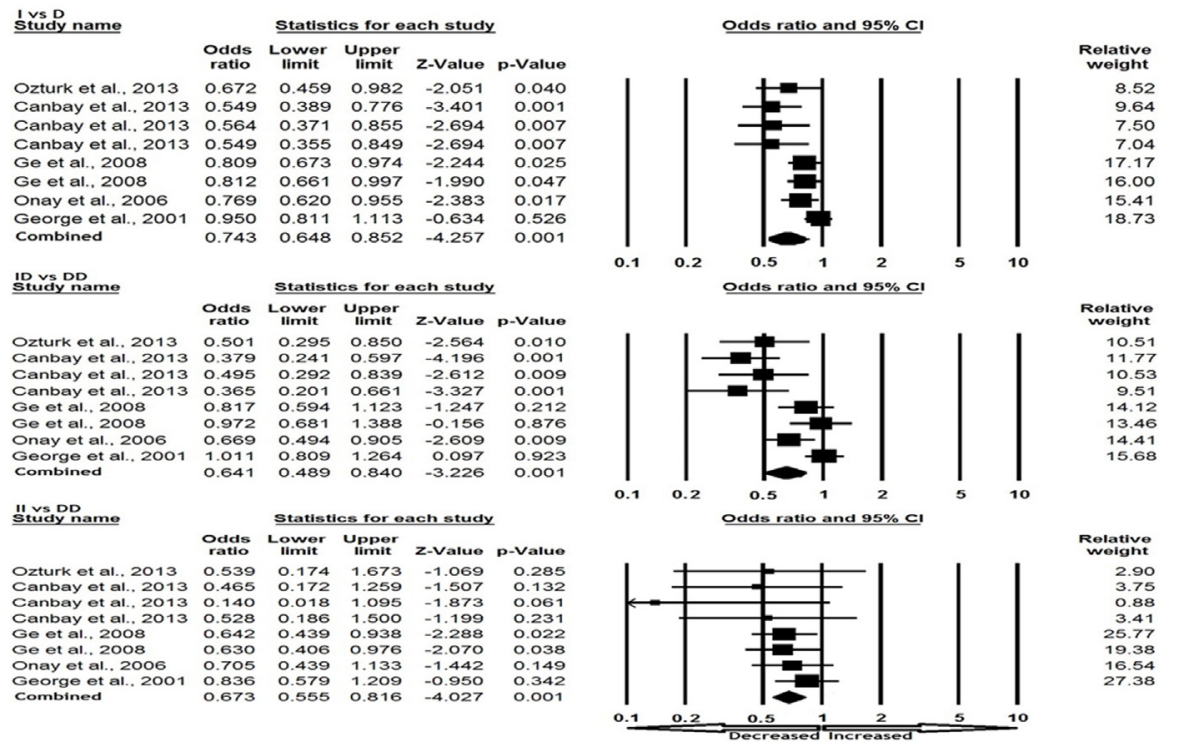

Figure 2. Forest Plot with ORs on Overall Cancer risk associated with PTEN IVS4 I/D gene polymorphism (I vs D; ID vs DD; II vs DD)

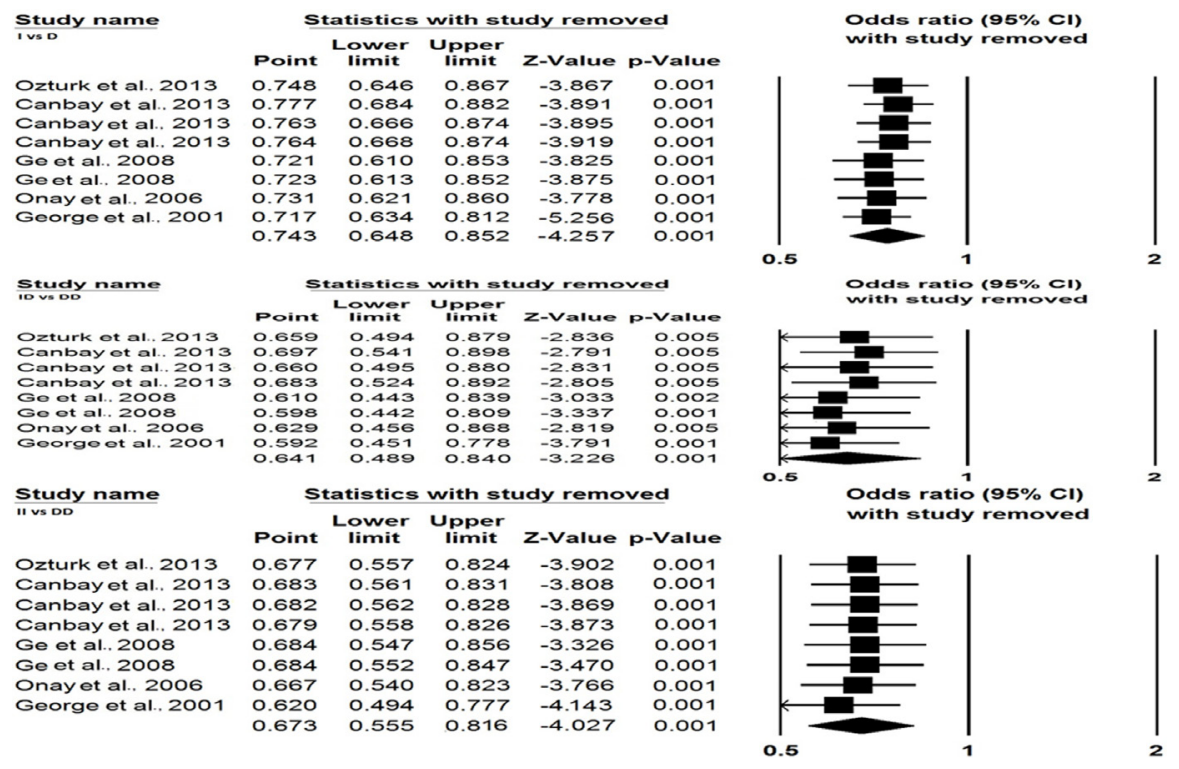

Figure 4. Sensitivity Analysis of PTEN IVS4 I/D Gene Polymorphism

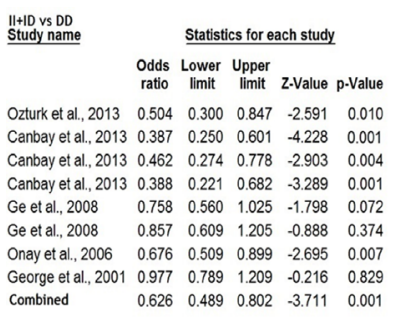

IVs DDD ID
Study name Statistics for each study

Odds Lower Upper
ratio
lilit

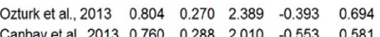
$\begin{array}{llllll}\text { Canbay et al., } 2013 & 0.760 & 0.288 & 2.010 & -0.553 & 0.581\end{array}$ $\begin{array}{lllllll}\text { Canbay et al., } 2013 & 0.204 & 0.027 & 1.574 & -1.524 & 0.127 \\ \end{array}$ $\begin{array}{lllllll}\text { Canbay et al. } 2013 & 0.838 & 0.306 & 2.296 & -0.343 & 0.732 \\ \text { Ge et al. 2008 } & 0.737 & 0.539 & 1.007 & -1.914 & 0.056\end{array}$ $\begin{array}{llllll}\text { Ge et al., 2008 } & 0.737 & 0.539 & 1.007 & -1.914 & 0.056 \\ \text { Ge et al. 2008 } & 0.642 & 0.448 & 0.921 & -2.410 & 0.016\end{array}$ $\begin{array}{llllll}\text { Ge et al., 2008 } & 0.642 & 0.448 & 0.921 & -2.410 & 0.016 \\ \text { Onay et al. 2006 } & 0.850 & 0.540 & 1.337 & -0.705 & 0.481\end{array}$ $\begin{array}{llllll}\text { Onay et al., 2006 } & 0.850 & 0.540 & 1.337 & -0.705 & 0.481 \\ \text { George et al. } 2001 & 0.832 & 0.586 & 1.180 & -1.032 & 0.302\end{array}$ $\begin{array}{llllll}\text { George et al., } 2001 & 0.832 & 0.586 & 1.180 & -1.032 & 0.302 \\ \text { Combined } & 0.749 & 0.631 & 0.889 & -3.311 & 0.001\end{array}$
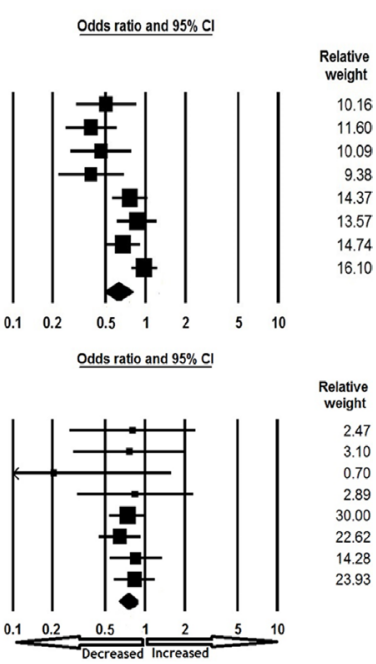

Figure 3. Forest Plot with ORs on Overall Cancer Risk Associated with PTEN IVS4 I/D Gene Polymorphism (II+ID $v s$ DD; II $v s$ DD+ID)

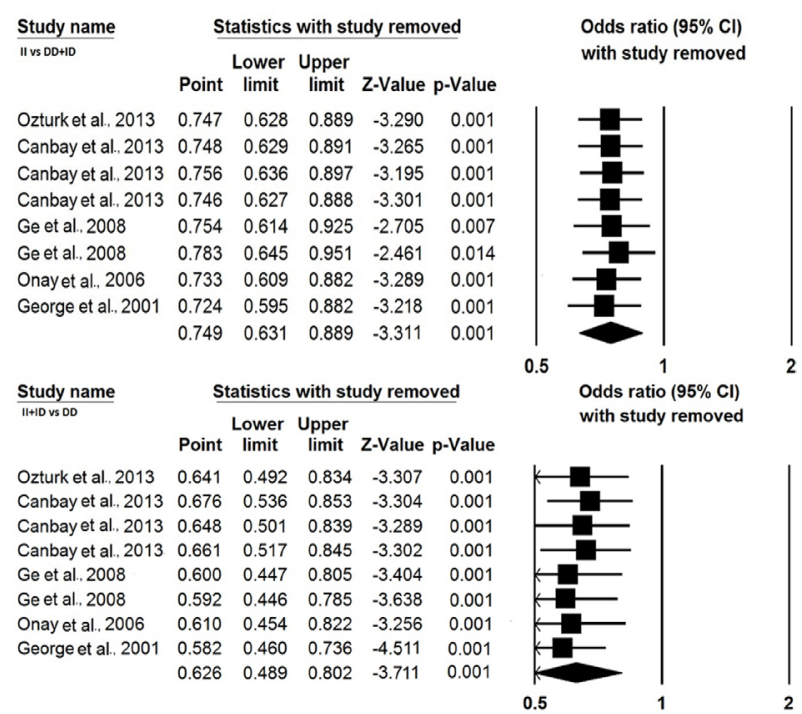

Figure 5. Sensitivity Analysis of PTEN IVS4 I/D Gene Polymorphism 
controls, and overall association between PTEN IVS4 I/D polymorphism and cancer risk was reviewed. The pooled OR from overall studies suggested association of decreased risk of cancer with PTEN IVS4 I/D polymorphism in allelic (I $v s$ D: $\mathrm{OR}=0.743,95 \% \mathrm{CI}=0.648$ to 0.852 , $\mathrm{p}=0.001$ ), homozygous (II $v s$ DD: $\mathrm{OR}=0.673$, $95 \% \mathrm{CI}=0.555$ to $0.816, \mathrm{p}=0.001$ ), heterozygous (ID vs $\mathrm{DD}: \mathrm{OR}=0.641,95 \% \mathrm{CI}=0.489$ to $0.840, \mathrm{p}=0.001)$, dominant (II+ID vs DD: $\mathrm{OR}=0.626,95 \% \mathrm{CI}=0.489$ to $0.802, \mathrm{p}=0.001)$ and recessive (II vs $\mathrm{DD}+\mathrm{ID}: \mathrm{OR}=0.749$, $95 \% \mathrm{CI}=0.631$ to $0.889, \mathrm{p}=0.001)$ genetic comparison models (Figure 2 and Figure 3 ).

\section{Sensitivity analysis}

We have performed sensitivity analysis by sequentially excluding individual study to evaluate the impact of single study included in the present study and to assess the stability of the present meta-analysis. The results of sensitivity analysis indicated that pooled OR before and after exclusion of each study were similar (Figure 4 and Figure 5). Hence, the results of the present meta-analysis are relatively stable and convincing.

\section{Discussion}

It has been widely accepted that genetic factors play an important role in the etiology of cancer and several low penetrance genes are involved in the progression of cancer. Therefore, genetic marker based risk assessment might offer some advantage to better predict the risk of cancer and early detection of the disease. PTEN participates in regulation of chromosomal integrity, acetylation of $\mathrm{p} 53$, DNA-damage response and the induction of apoptosis (Salmena et al., 2008), and variations in PTEN expression play an important role in the regulation of cell proliferation and tumor invasion (Zhou et al., 2003). Recently, a moderate decrease in PTEN expression to $80 \%$ of the normal level has been revealed to increase susceptibility to develop cancer in mice (Alimonti et al., 2010).

Considering the significance of PTEN gene in carcinogenesis, it is rational to speculate that host genomic polymorphism of PTEN gene may affect the tumor development (Mahdi et al, 2013). In last few years, the genetic variants of the PTEN including IVS4 I/D and its possible role in the etiology of several cancers have been studied extensively, but the results are inconsistent and inconclusive. It is well recognized that the individual studies with a small sample size may have not enough statistical power to detect a small risk factor. In the present study, our main focus was to estimate the more precise results to understand the contribution of PTEN IVS4 I/D gene polymorphism in overall cancer risk. Therefore, we performed the current meta-analysis to assess whether PTEN IVS4 I/D gene polymorphism could have an impact on the susceptibility to overall cancer.

To the best of our knowledge, our meta-analysis represents the first pooled data study investigating the association between PTEN IVS4 I/D gene polymorphism and overall cancer risk. The associations for the allele contrast, dominant and recessive model were examined in the meta-analysis. Statistical analysis revealed that the PTEN IVS4 I/D polymorphism is associated with a significantly decreased susceptibility to overall cancer. PTEN IVS4 polymorphism and susceptibility to cancer is diverged and previous studies demonstrated that DD genotype of PTEN IVS4 was found to be associated with early onset of breast cancer and male cases of esophageal and gastric cardia cancer (Carroll et al., 2000; Ge et al., 2008). On the other hand, it was correlated with reducing incidence of high grade/stage of prostate cancer among cases younger than 69 years old (George et al., 2001). Genetically complex diseases differ from simple Mendelian diseases and cancer etiology is polygenic, a single genetic variant is usually inadequate to predict the risk of this deadly disease that has a complex disease phenotype. It is well established that gene polymorphisms are complicated and fluctuating, which is mainly attributed to different ethnicities. The relationship between cancer susceptibility and variant genotypes of PTEN IVS4 might be affected by the tumor sites.

Though, we interpreted our findings with full caution, but, this meta-analysis still had some limitations. Heterogeneity is an important issue while interpreting the results of meta-analysis, although, it can be minimized by applying random-effects model (Munafo et al., 2004). In the present study we detected inter-study heterogeneity in three genetic models. The source of heterogeneity may arise possibly due to, region of study, the sample size, the case and the control group, clinical characteristics of different tumors, and the genotyping method. Due to the limited number of published studies, subgroup analysis was not performed and the current meta-analysis was based on unadjusted estimates, while a more precise analysis could be performed if individual data were available. In spite of this, we used explicit criteria for a study's inclusion and performed strict data extraction and analysis to make satisfactory and reliable conclusion.

In conclusion, the results of this meta-analysis indicated that the PTEN IVS4 I/D gene polymorphism is associated with decreased risk of cancer. In the present study, we only analyzed the IVS4 I/D variant without considering the interaction between several other SNPs and in our future study we will try to explore the other pertinent interactions to facilitate the discovery of the pathogenesis of cancer. Our study also warrants for well-designed large-scale studies with consideration of gene-gene and gene-environment interactions to further evaluate this possible association..

\section{Acknowledgements}

Authors sincerely acknowledge the software program related service support for statistical analysis provided by the Institute of Life Sciences, Bhubaneswar, India and Jamia Millia Islamia (Central University) New Delhi, India.

\section{References}

Alimonti A, Carracedo A, Clohessy JG, et al (2010). Subtle variations in PTEN dose determine cancer susceptibility. Nat Genet, 42, 454-8. 


\section{Raju K Mandal et al}

Besson A, Robbins SM, Yong VW (1999). PTEN/MMAC1/TEP1 in signal transduction and tumorigenesis. Eur J Biochem, 263, 605-11.

Canbay E, Kahraman OT, Bugra D, et al (2013a). Association between PTEN IVS4 polymorphism and development of colorectal cancer in a Turkish population. Expert Opin Ther Targets, 17, 1-6.

Canbay E, Kahraman OT, Bugra D, et al (2013b). Increased gastric cancer risk with PTEN IVS4 polymorphism in a Turkish population. Genet Test Mol Biomarkers, 17, 249-53.

Carroll BT, Couch FJ, Rebbeck TR, et al (2000). Polymorphisms in PTEN in breast cancer families. J Med Genet, 36, 94-96.

Cheng T, Zhang J, Cheng Y, et al (2012). Relationship between PTEN and livin expression and malignancy of renal cell carcinomas. Asian Pac J Cancer Prev, 13, 2681-5

Cohn LD, Becker BJ (2003). How meta-analysis increases statistical power. Psychol Methods, 3, 243-53.

DerSimonian R, Laird N (1986). Meta-analysis in clinical trials. Control Clin Trials, 7, 177-188.

Duval S, Tweedie R (2000). Trim and fill: a simple funnelplotbased method of testing and adjusting for publication bias in meta-analysis. Biometrics, 56, 455-63.

Egger M, Davey Smith G, Schneider M, et al (1997). Bias in meta-analysis detected by a simple, graphical test. $B M J$, 7109, 629-34.

Ge H, Cao YY, Chen LQ, et al (2008). PTEN polymorphisms and the risk of esophageal carcinoma and gastric cardiac carcinoma in a high incidence region of China. Dis Esophagus, 21, 409-15.

George DJ, Shepard TF, Ma J, et al (2001). PTEN polymorphism (IVS4) is not associated with risk of prostate cancer. Cancer Epidemiol Biomarkers Prev, 10, 411-412.

Higgins JP, Thompson SG, Deeks JJ, et al (2003). Measuring inconsistency in meta-analyses. $B M J, \mathbf{7 4 1 4}, 557-60$.

Jemal A, Bray F, Center MM, et al (2011). Global cancer statistics. CA Cancer J Clin, 61, 69-90.

Jia-Li X, Zhen-Wu W, Ling-Min H, et al (2012). Genetic variants in the PI3K/PTEN/AKT/mTOR pathway predict platinumbased chemotherapy response of advanced non-small cell lung cancers in a Chinese population. Asian Pac J Cancer Prev, 13, 2157-62.

Li DM, Sun H (1997). TEP1, encoded by a candidate tumor suppressor locus, is a novel protein tyrosine phosphatase regulated by transforming growth factor beta. Cancer Res, 57, 2124-9.

Li J, Yen C, Liaw D, Podsypanina K, et al (1997). PTEN, a putative protein tyrosine phosphatase gene mutated in human brain, breast, and prostate cancer. Science, 275, 1943-1947.

Lichtenstein P, Holm NV, Verkasalo PK, et al (2000). Environmental and heritable factors in the causation of cancer-analyses of cohorts of twins from Sweden, Denmark, and Finland. N Engl J Med, 343, 78-85.

Mahdi KM, Nassiri MR, Nasiri K (2013). Hereditary Genes and SNPs associated with breast cancer. Asian Pac J Cancer Prev, 14, 3403-9

Mandal RK, Yadav SS, Panda AK (2013). Meta-analysis on the association of nucleotide excision repair gene XPD A751C variant and cancer susceptibility among Indian population. Mol Biol Rep, 41, 713-9.

Mantel N, Haenszel W (1959). Statistical aspects of the analysis of data from retrospective studies of disease. J Natl Cancer Inst, 4, 719-48.

Munafò MR, Flint J (2004). Meta-analysis of genetic association studies. Trends Genet, 20, 439-444.

Onay VU, Briollais L, Knight JA, et al (2006). SNP-SNP interactions in breast cancer susceptibility. BMC Cancer, 6, 114 .
Ozturk O, Canbay E, Kahraman OT, et al (2013). HER2 Ile655Val and PTEN IVS4 polymorphisms in patients with breast cancer. Mol Biol Rep, 40, 1813-8.

Pandurangan AK (2013). Potential targets for prevention of colorectal cancer: a focus on PI3K/Akt/mTOR and Wnt pathways. Asian Pac J Cancer Prev, 14, 2201-5

Pharoah PD, Dunning AM, Ponder BA, et al (2004). Association studies for finding cancer-susceptibility genetic variants. Nat Rev Cancer, 4, 850-60.

Salmena L, Carracedo A, Pandolfi PP (2008). Tenets of PTEN tumor suppression. Cell, 133, 403-14.

Steck PA, Pershouse MA, Jasser SA, et al (1997). Identification of a candidate tumour suppressor gene, MMAC1, at chromosome 10q23.3 that is mutated in multiple advanced cancers. Nat Genet, 15, 356-62.

Sun H, Lesche R, Li DM, et al (1999). PTEN modulates cell cycle progression and cell survival by regulating phosphatidylinositol 3,4,5,-trisphosphate and Akt/protein kinase B signaling pathway. Proc Natl Acad Sci USA, 96, 61996204.

Tamura M, Gu J, Matsumoto K, et al (1998). Inhibition of cell migration, spreading, and focal adhesions by tumor suppressor PTEN. Science, 280, 1614-7.

Tamura M, Gu J, Takino T, et al (1999). Tumor suppressor PTEN inhibition of cell invasion, migration, and growth: differential involvement of focal adhesion kinase and p130Cas. Cancer Res, 59, 442-9.

Wen-Ting X, Zhen Yang, Nong-Hua Lu (2014) Roles of PTEN (phosphatase and tensin homolog) in gastric cancer development and progression. Asian Pac J Cancer Prev, 15, 17-24.

Woolf B (1955). On estimating the relation between blood group and disease. Ann Hum Genet, 19, 251-3.

Wu R, Li B (1999). A multiplicative-epistatic model for analyzing interspecific differences in outcrossing species. Biometrics, 2, 355-65.

Wu X, Senechal K, Neshat MS, et al (1998). The PTEN/MMAC1 tumor suppressor phosphatase functions as a negative regulator of the phosphoinositide 3-kinase/Akt pathway. Proc Natl Acad Sci USA, 95, 15587-91.

Zhou YH, Tan F, Hess KR, et al (2003). The expression of PAX6, PTEN, vascular endothelial growth factor, and epidermal growth factor receptor in gliomas: relationship to tumor grade and survival. Clin Cancer Res, 9, 3369-75. 